\title{
Noise Figure Evaluation of Polarization-insensitive Single- pump Fiber Optical Parametric Amplifiers
}

\author{
Vladimir Gordienko ${ }^{{ }^{*}}$, Filipe Ferreira ${ }^{1}$, Charles Laperle ${ }^{2}$, Maurice O'Sullivan ${ }^{2}$, Chandra B Gaur ${ }^{1}$, \\ Kim Roberts ${ }^{2}$, and Nick Doran ${ }^{1}$ \\ ${ }^{1}$ Aston Institute of Photonic Technologies, Aston University, Birmingham, B4 7ET, UK \\ ${ }^{2}$ Ciena Corporation, Ottawa, Ontario K2K 0L1, Canada \\ *gordiev1@aston.ac.uk
}

\begin{abstract}
Several polarization-insensitive configurations for single-pump phase-insensitive fiber optical parametric amplifier are experimentally evaluated using 35GBaud PDM-QPSK signals. An equivalent noise figure of $9.1 \pm 1 \mathrm{~dB}$ is experimentally derived by comparison with a variable noise figure EDFA. C 2020 The Authors. OCIS codes: (060.2320) Fiber optics amplifiers and oscillators; (190.4970) Parametric oscillators and amplifiers.
\end{abstract}

\section{Introduction}

Fiber optical parametric amplifiers (FOPA) feature theoretically unconstrained operation wavelength range and a possibility of high gain over wide bandwidth [1]. This makes FOPA an attractive amplifier for emerging ultra-wide band communication. In addition, lack of transient effects makes FOPA appealing for extended PON with burst traffic [2]. Finally, FOPA are capable of phase sensitive amplification enabling noise figure as low as $1.1 \mathrm{~dB}$ [3].

Recent advances in polarization-insensitive FOPA [4] have enabled amplification of polarization multiplexed signals [5] and multi-span transmission experiments [6]. These experiments have utilized half-pass loop (HPL) architecture. In HPL-FOPA an arbitrary polarization signal is split into two linearly polarized components propagating in opposite directions within the loop. Signal components are equally amplified by corresponding copropagating pumps in highly nonlinear fiber (HNLF). HPL-FOPA employs at least two sections of HNLF each amplifying only one signal component. Therefore, each signal component passes through 'Gain' (where it is amplified) and 'Loss' (where it experiences passive loss, while the other component is amplified) sections of HNLF.

Three configurations of HPL-FOPA have been experimentally demonstrated so far. Two of them differ in the order of the 'Gain' and the 'Loss' sections. They are called: 'Gain - Loss' (GL) and 'Loss - Gain' (LG). Another configuration 'Gain - Filter - Loss' (GFL) features an additional filter to remove idlers. GL has been shown to suffer from significant unwanted four wave mixing (FWM) products arising in the 'Loss' section. LG and GFL have been shown to produce $\sim 10 \mathrm{~dB}[7]$ and $\sim 4.5 \mathrm{~dB}$ [8] less unwanted FWM products power respectively than GL. However, an additional linear noise in LG has been shown to introduce $\mathrm{Q}^{2}$ penalty of $\sim 1 \mathrm{~dB}$ as compared to GL [9].

In this paper for the first time to the best of our knowledge we evaluate the noise figure (NF) of polarizationinsensitive FOPA. We measure the NF for each HPL-FOPA configuration and find a minimum NF of $5.8 \pm 1 \mathrm{~dB}$. Then we compare performance of HPL-FOPA configurations with each other and against an EDFA with a varied $\mathrm{NF}$ in terms of amplified signal $\mathrm{Q}^{2}$ derived from error counting. We find that the conventional NF measurement fails to account for FOPA specific impairments such as the pump dithering transfer, signal self-phase modulation and polarization-dependent gain (PDG). We find that EDFA with NF of $9.1 \pm 1 \mathrm{~dB}$ allows for $\mathrm{Q}^{2}$ similar to that of the FOPA with the NF of $5.8 \mathrm{~dB}$. We then discuss means to obtain a commercial EDFA equivalent performance.

\section{Experimental setup}

Fig. 1 shows an experimental setup to evaluate HPL-FOPA configurations in terms of NF and BER and to compare them with EDFA. The setup consists of WDM signals emulator, WDM signals receiver and an amplifier under test. Five amplification scenarios have been tested: three HPL-FOPA configurations and two EDFA scenarios. One EDFA scenario had a $5 \mathrm{~dB}$ attenuation after the EDFA to achieve low NF, whilst another scenario had a $5 \mathrm{~dB}$ attenuation before the EDFA to degrade its NF. Net gain of all configurations and scenarios was $14 \mathrm{~dB}$.

The WDM signals emulator produced 21x 50GHz-spaced channels between $1533.5 \mathrm{~nm}$ and $1541.3 \mathrm{~nm}$. The middle channel at $1537.4 \mathrm{~nm}$ was 35 GBaud PDM-QPSK (100G) signal sourced from a Ciena transponder WaveLogic 3. The remaining channels were sourced by from ASE noise using a wavelength selective switch (WSS) to replicate the $100 \mathrm{G}$ channel spectral shape. An EDFA followed by a variable optical attenuator (VOA) were then employed to set a power per channel in range from $-25 \mathrm{dBm}$ to $-4 \mathrm{dBm}$.

At the receiver side the amplifier output was coupled with ASE noise using a 5\% coupler. A WSS separated the $100 \mathrm{G}$ channel from other channels and attenuated it to $-19 \mathrm{dBm}$. Then, the $100 \mathrm{G}$ channel was coherently detected by the Ciena transponder WaveLogic 3. 
All HPL-FOPA configurations are shown at Fig. 2. First, the input signal was split into orthogonal linearly polarized components using a polarization beam splitter (PBS). Each signal component was amplified in one of two lengths $(250 \mathrm{~m}$ and $200 \mathrm{~m}$ ) of dispersion-stable HNLF by a co-propagating pump. HNLF parameters were zerodispersion wavelength of $\sim 1564 \mathrm{~nm}$, dispersion slope of $84 \mathrm{~s}^{-3} \mathrm{~m}^{-3}$ and nonlinear coefficient of $8.2 \mathrm{~W}^{-1} \cdot \mathrm{km}^{-1}$. WDM filters were placed at the ends of HNLF lengths to add/drop pumps. In the GL and the GFL configurations the pumps were injected in the HNLF lengths via WDMs near the PBS to amplify signal components once they enter the loop. In the LG configuration the pumps were injected into the HNLF lengths via WDMs in the middle of the loop to amplify signal components as they passed through their second HNLF length. Pumps at the wavelength of $1564.4 \mathrm{~nm}$ were sourced from a $100 \mathrm{kHz}$ linewidth laser, phase modulated to mitigate stimulated Brillouin scattering and amplified by high power EDFAs. Pump powers were tuned independently to ensure the $100 \mathrm{G}$ channel net gain of $14 \pm 0.2 \mathrm{~dB}$ and $\mathrm{PDG}<0.2 \mathrm{~dB}$ in every measurement. Pump powers in the $250 \mathrm{~m}$ and the $200 \mathrm{~m}$ lengths of HNLF were in ranges of $1.9 \ldots 2.4 \mathrm{~W}$ and $2.4 \ldots .2 .9 \mathrm{~W}$ respectively across all measurements. In the GFL configuration two $\mathrm{C} / \mathrm{L}$ band splitters were added to remove idlers of amplified signal components.

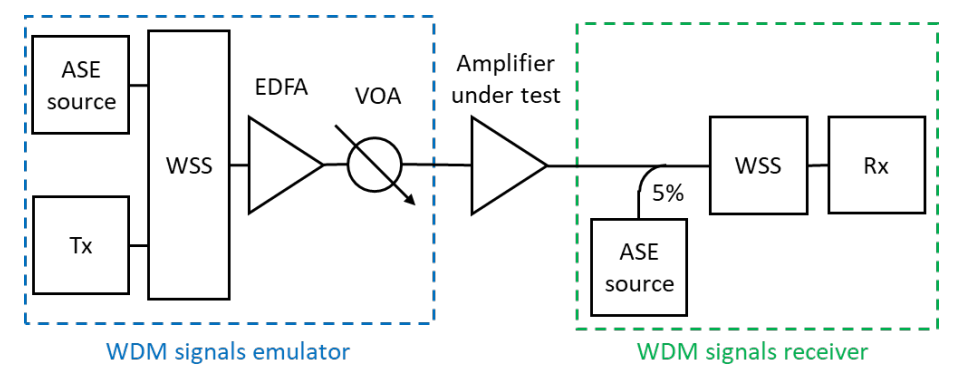

Fig. 1. Experimental setup to evaluate HPL-FOPA configurations.
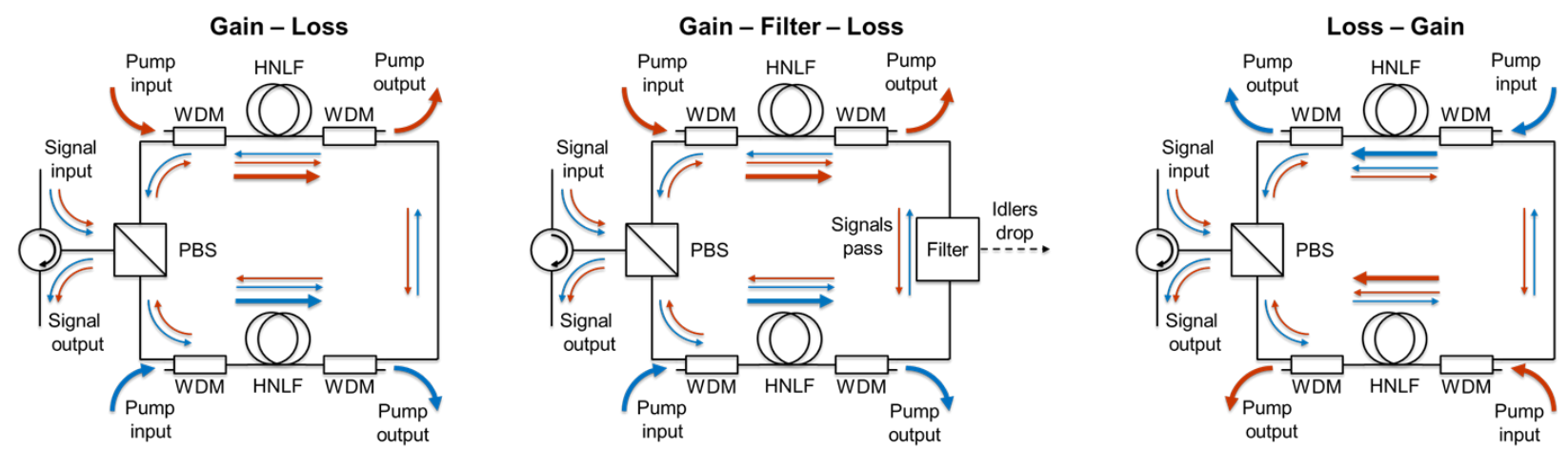

Fig. 2. Examined HPL-FOPA configurations.

\section{Results and discussion}

The NF was calculated using Eq. (1) [10], where $N_{\text {input }}$ and $N_{\text {output }}$ were noise powers measured using an optical spectrum analyzer (OSA) via calibrated tap couplers at the input and the output of a tested amplifier, $B$ was the OSA resolution bandwidth equal $19 \mathrm{GHz}, v$ was the $100 \mathrm{G}$ channel frequency of $195 \mathrm{THz}$, and $G$ was the net gain. The $100 \mathrm{G}$ channel was rejected by the WDM signals emulator WSS to measure noise powers. In the EDFA scenarios the power of the adjacent channels was increased to account for the rejected channel. By applying this formulation to FOPA, the NF measurements include the unwanted FWM products power as a part of the noise power. The NF measurement error was estimated to be less than $1 \mathrm{~dB}$.

$$
N F=\frac{N_{\text {output }}}{G h v B}+\frac{1}{G}-\frac{N_{\text {input }}}{h v B}
$$

Figure 3(a) shows a minimum NF of $5.8 \mathrm{~dB}$ and $6.5 \mathrm{~dB}$ for GFL and GL configurations respectively. However, as signal power increases, so does that of unwanted FWM, and the NF grows by up to $3 \mathrm{~dB}$ per $1 \mathrm{~dB}$ of signal power increase. The LG configuration had a minimum NF of $11.8 \mathrm{~dB}$, much higher than GL and GFL. Nevertheless, the LG configuration robustness against unwanted FWM allows for lower NF than GL and GFL configurations as signal power increases. It should be noted that there is a large scope to improve the measured FOPA NF. For instance, the FOPA NF in linear regime is essentially $3 \mathrm{~dB}$ plus upfront loss, so the excess loss optimization in the HPL should 
enable linear regime NF of $\sim 4 \mathrm{~dB}$. Additionally, power of unwanted FWM products scales as $1 / L^{2}$, where $L$ is the HNLF length, so if we were to use $50 \mathrm{~m}$ long HNLFs that would allow for $12 \mathrm{~dB}$ higher signal power without the NF being degraded by the unwanted FWM.

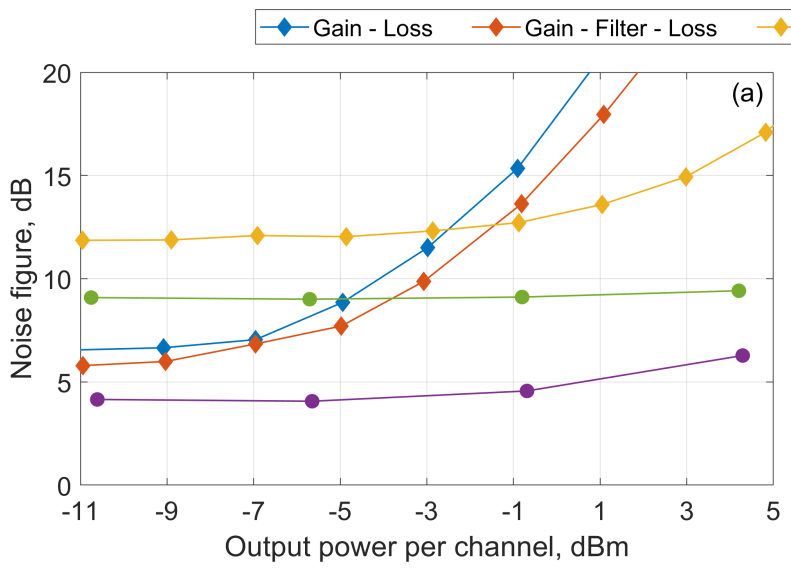

Loss - Gain $\rightarrow$ EDFA (low NF) $\longrightarrow$ EDFA (high NF)

Fig. 3. (a) Noise figure and (b) $Q^{2}$ measured for each HPL-FOPA configuration and two EDFA scenarios.

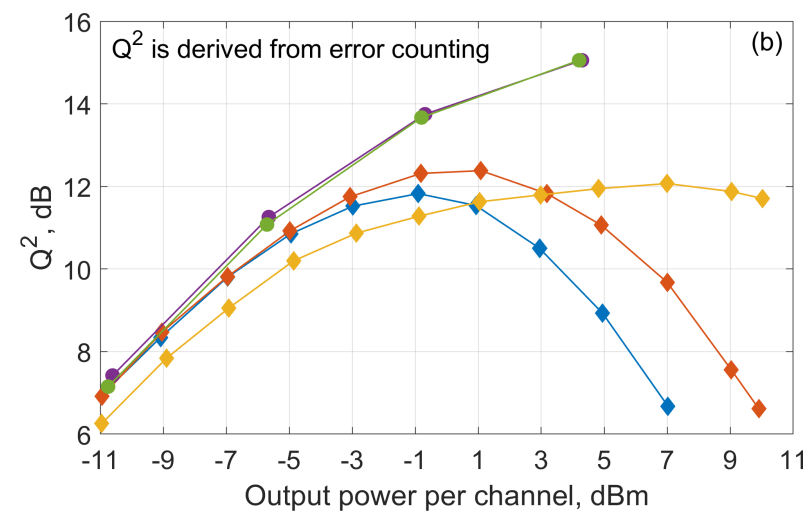

Figure 3(b) shows $\mathrm{Q}^{2}$ derived from the error counting for all examined scenarios. In accordance with the NF measurements the LG configuration has $\sim 1 \mathrm{~dB}$ lower $\mathrm{Q}^{2}$ than the GL and the GFL configurations at low signal power. As signal power increases the $\mathrm{Q}^{2}$ degrades quickly first in the GL and then in the GFL configuration with the LG eventually outperforming the other two configurations. An important observation is that the GL FOPA with the $\mathrm{NF}$ of $5.8 \pm 1 \mathrm{~dB}$ performs rather similar to the EDFA scenario with NF of $9.1 \pm 1 \mathrm{~dB}$. This is because of other impairments not captured by the NF measurement: pump dithering transfer to signals, self-phase modulation and the residual PDG. Mitigation of these effects by optimization of dithering, an HNLF length decrease and minimization of PDG in HPL-FOPA through active loop control along with the HPL excess loss optimization should enable FOPA to achieve a commercial EDFA equivalent performance whilst delivering its advantages of wider bandwidth, arbitrary wavelength range operation and instantaneous response time.

\section{Conclusion}

We demonstrate the ability of the HPL architecture for polarization insensitive FOPA to provide a competitive NF of $5.8 \pm 1 \mathrm{~dB}$. We observe such a FOPA to perform similarly to an EDFA with the NF of $\sim 9.1 \pm 1 \mathrm{~dB}$. We propose the difference between the FOPA own NF and the EDFA equivalent NF is due to pump dithering transfer, self-phase modulation and the residual PDG. We conclude that having these impairments mitigated and the own FOPA NF optimized there is a realistic expectation to obtain a commercial EDFA equivalent performance with HPL-FOPA.

\section{Acknowledgements}

The work was funded by EPSRC projects UPON (EP/M005283/1) and FPA-ROCS (EP/R024057/1). The data reported in this paper is available at https://doi.org/10.17036/researchdata.aston.ac.uk.00000442.

\section{References}

[1] M. E. Marhic, Fiber Optical Parametric Amplifiers, Oscillators and Related Devices, (Cambridge University Press, New York, 2008).

[2] C. B. Gaur, F. Ferreira, V. Gordienko, et al, "Demonstration of improved performance provided by FOPA for extended PON in burst-mode operation," in European Conference on Optical Communications (ECOC), Dublin, Ireland, Sep. 2019, paper Th.1.C.4.

[3] Z. Tong, C. Lundström, P. A. Andrekson, et al, “Towards ultrasensitive optical links enabled by low-noise phase-sensitive amplifiers," Nat. Photonics, vol. 5, no. 7, June 2011, pp. 430-436.

[4] S. Takasaka and R. Sugizaki, "Polarization insensitive fiber optical parametric amplifier using a SBS suppressed diversity loop," in Optical Fiber Communication Conference (OFC), Anaheim, CA, March 2016, paper M3D.4.

[5] M. F. C. Stephens, V. Gordienko, and N. J. Doran, "20 dB net-gain polarization -insensitive fiber optical parametric amplifier with $>2 \mathrm{THz}$ bandwidth," Opt. Express, vol. 25, no. 9, May 2017, pp. 10597-10609.

[6] M. F. C. Stephens, M. Tan, V. Gordienko, et al, "In-line and cascaded DWDM transmission using a 15dB net-gain polarization-insensitive fiber optical parametric amplifier," Opt. Express, vol. 25, no. 20, Oct. 2017, pp. 24312-24325.

[7] M. F. C. Stephens, V. Gordienko, and N. J. Doran, "Reduced crosstalk, polarization insensitive fiber optical parametric amplifier (PI FOPA) for WDM applications," in Optical Fiber Communications Conference (OFC), San Diego, CA, March 2018, paper W3D.4.

[8] V. Gordienko, F. Ferreira, V. Ribeiro, et al, "Suppression of Nonlinear Crosstalk in a Polarization Insensitive FOPA by Mid-stage Idler Removal," in Optical Fiber Communication Conference (OFC), San Diego, CA, March 2019, paper M4C.4.

[9] V. Gordienko, F. Ferreira, A. Szabo, et al, "Characterisation of Novel Polarisation-Insensitive Configurations of Fibre Optical Parametric Amplifiers," in European Conference on Optical Communications (ECOC), Dublin, Ireland, Sep. 2019, paper Th.1.C.5.

[10] D. M. Baney, P. Gallion, and R.S. Tucker, "Theory and Measurement Techniques for the Noise Figure of Optical Amplifiers," Optical Fiber Technology 6, 122-154 (2000). 